Published in final edited form as:

Trends Cell Biol. 2016 March ; 26(3): 202-214. doi:10.1016/j.tcb.2015.10.002.

\title{
Making a Hematopoietic Stem Cell
}

\author{
Michael G. Daniel ${ }^{1,2,3}$, Carlos-Filipe Pereira ${ }^{4}$, Ihor R. Lemischka ${ }^{1,2,5}$, and Kateri A. \\ Moore ${ }^{1,2,{ }^{*}}$ \\ ${ }^{1}$ Department of Developmental and Regenerative Biology, Icahn School of Medicine, New York, \\ NY, USA \\ ${ }^{2}$ Black Family Stem Cell Institute, Icahn School of Medicine, New York, NY, USA \\ ${ }^{3}$ The Graduate School of Biomedical Science, Icahn School of Medicine, New York, NY, USA \\ ${ }^{4} \mathrm{CNC}$ - Center for Neuroscience and Cell Biology, University of Coimbra, UC Biotech Building, \\ Biocant Park, 3060-197 Cantanhede, Portugal \\ ${ }^{5}$ Department of Pharmacology and Systems Therapeutics, Icahn School of Medicine, New York, \\ NY, USA
}

\begin{abstract}
Previous attempts to either generate or expand hematopoietic stem cells (HSCs) in vitro have involved either ex vivo expansion of pre-existing patient or donor HSCs or de novo generation from pluripotent stem cells (PSCs), comprising both embryonic stem cells (ESCs) and induced pluripotent stem cells (iPSCs). iPSCs alleviated ESC ethical issues but attempts to generate functional mature hematopoietic stem and progenitor cells (HSPCs) have been largely unsuccessful. New efforts focus on directly reprogramming somatic cells into definitive HSCs and HSPCs. To meet clinical needs and to advance drug discovery and stem cell therapy, alternative approaches are necessary. In this review, we synthesize the strategies used and the key findings made in recent years by those trying to make an HSC.
\end{abstract}

\section{The Need for Patient-Specific HSPCs and Strategies to Obtain Them}

Hematopoiesis (see Glossary), the process by which hematopoietic stem cells (HSCs) generate all the cellular elements in our blood, established the paradigm for stem cell therapy. It proceeds in a hierarchical manner anchored by self-renewing HSCs. They give rise to progenitors with limited self-renewal potential that differentiate into lineage-restricted cells, making up the immunohematopoietic system. Source material for hematopoietic transplantation is in great demand as at least 20000 allogeneic transplants are performed every year [1]. Despite advances in using umbilical cord blood (UCB) and mobilized stem cells, donor material remains restricted by limited stem cells in UCB, poor mobilization, and the lack of ethnic diversity to provide sufficiently matched material [2]. Allogeneic transplants require donor and host human leukocyte antigen (HLA) matching, and can cause graft-versus-host disease (GvHD) and graft rejection [3].

\footnotetext{
'Correspondence: kateri.moore @mssm.edu (K.A. Moore).
} 
To overcome the aforementioned challenges, some studies have sought to expand HSPC numbers in vitro through the expansion of ex vivo HSPCs with small molecules. Success has been reported using SR1, UM171, and valproic acid [4-6]. Although small molecules have demonstrated utility in somatic cell reprogramming strategies such as fibroblasts to cholinergic neurons and others, their use with hematopoietic cells is still limited [7,8]. Despite their ease of optimization experimentally, various side effects have been reported when using small molecules [9,10], and there remain limitations in both the overall function of the expanded HSPCs and who can be treated with them. For these reasons, alternative sources of transplantable allogeneic and patient-specific HSCs are required.

A paradigm shift in stem cell biology - and the beginning of the field of regenerative medicine -occurred when Yamanaka and Takahashi reprogrammed somatic cells to iPSCs using four transcription factors (TFs) [11,12]. Further understanding of transcriptional control in a number of different cell types [13] has expanded the use of TFs to directly change somatic cell fates without going through pluripotency $[14,15]$. Indeed, progress has been made in reprogramming fibroblasts to other cell types such as monocyte-like progenitor cells, macrophages, and angioblast-like progenitor cells, among others [16-29], but few attempts have been made at reprogramming somatic cells into a stem cell with the degree of multipotency that an HSC possesses [30]. This possibility makes the de novo generation of HSCs from patient-specific cells a major goal of regenerative medicine: patient cells would be harvested, genetically corrected, reprogrammed, expanded in vitro, and used for autologous HSC transplant [31,32]. Having these cells to study in vitro would also permit drug discovery for a range of different disorders and allow insights into the transcriptional control of hematopoiesis (Figure 1).

After decades of research, differentiating PSCs into engraftable multilineage HSCs has largely been unsuccessful [33]. Multiple studies, however, bring us much closer to such a coveted feature of regenerative stem cell biology (Table 1), which is the focus of this review. Moreover, advances in 'omics' technology and the direct conversion of somatic cells to an HSC state may soon make this aspect of regenerative stem cell biology a viable option.

\section{Differentiating ESCs and iPSCs to Bona Fide Definitive HSCs}

The first endeavors to generate HSCs and other progenitor cells in vitro arose from PSC hematopoietic differentiation [34,35]. Efforts using PSCs, however, have not yielded robust results because of limited multilineage long-term engraftment potential [36,37]. It is thought that PSC-derived hematopoietic cells do not fully mature to an adult stage. These cells do not effectively give rise to cells of all lineages and fail to produce adult hemoglobin, nor do they home to the bone marrow effectively.

\section{Recapitulating Hematopoietic Development with PSCs}

Potential HSCs were first seen in vitro emerging from embryoid bodies (EBs) via ESC differentiation upon cytokine supplementation [37,38]. Later efforts focused on recapitulating embryonic hematopoietic development by differentiating PSCs. PSCs can now be differentiated into hemogenic endothelium (HE), the progenitor cell population theorized to give rise to HSCs as part of an embryonic site of hematopoiesis called the aorta- 
gonad-mesonephros [39-42]. Recent data demonstrate that different populations of HE give rise to the primitive and definitive hematopoietic programs within these hematopoietic sites [43]. Primitive hematopoiesis emerges first during development, with cells possessing a transient nature and restricted potential (erythrocytes, macrophages, and megakaryocytes). By contrast, the definitive program contains HSCs that develop from HE via an endothelialto-hematopoietic transition (EHT) [44]. Runx1, among other TFs, is crucial for this early transition process [45]. These two programs are typically studied using T-lymphoid potential as a read-out [46], but this has been recently challenged because immune cells were found to emerge prior to definitive hematopoiesis [47]. Moreover, hemangioblasts (HBs) from mouse PSCs form through an HE precursor to reach the primitive program, complicating our ability to tease apart the different HE populations, and thus our ability to easily generate HSCs [48].

Recently, several groups have investigated using teratomas as sources of HSCs. Teratomas contain tissues from all three germ layers, suggesting that they would also contain cells with inductive signals of the hematopoietic niche to induce formation of HSCs. Human iPSCs injected into immunocompromised mice generated teratomas that contained hematopoietic cells. HSPCs isolated from these teratomas could be serially transplanted and restore the hematopoietic system of immunocompromised mice [49]. iPSCs derived from $\mathrm{Lnk}^{-/}$mice have high hematopoietic potential. This adaptor protein is normally expressed in hematopoietic progenitors and inhibits c-Kit-mediated proliferation, regulating expansion and function of hematopoietic progenitors [50]. Therefore, removal of Lnk will increase the yield of hematopoietic cells in this strategy, and also possibly permit further study into the mechanisms of Lnk signaling to control hematopoietic progenitor expansion from HSCs. $\mathrm{Lnk}^{-/}$iPSCs, engineered to express the common gamma chain protein, were used to induce teratomas from which HSPCs were isolated and used to correct X-SCID mice, which carried the common gamma chain mutation after transplantation [51]. These approaches are limited in their clinical use due to the risk of reforming teratomas, but provide a reproducible strategy to study the molecular mechanisms of various signaling pathways in HSC biology.

\section{Directed Differentiation of PSCs with TF Reprogramming}

Additional efforts with PSCs have looked to overexpression of critical hematopoietic TFs. The homeobox gene HoxB4 has multiple roles in hematopoiesis and was the first to be overexpressed [52]. Early attempts employed retroviruses to introduce HoxB4 ectopically into mouse embryonic stem cells (mESCs). After culture on OP9 stroma these cells were used to rescue lethally irradiated mice but primarily adopted a myeloid fate [53]. Inducible HoxB4 and OP9 co-culture also led to in vitro generation of HSPCs from mESCs (Figure 2). These precirculation yolk sac and ESC-derived progenitors possess multilineage engraftment potential in irradiated adult primary and secondary recipient mice. Long-term constitutive over-expression of HoxB4, however, eventually inhibits differentiation [54]. As a result of genomic integration of HoxB4, it is unlikely that this technique could be applied clinically; therefore, attempts have been made to package HoxB4 in adenovirus to avoid viral integration. Transient HoxB4 overexpression allows for HSPC generation from mouse iPSCs [55], but it remains unclear if the derived cells are capable of long-term engraftment. Inducible overexpression of $\mathrm{Cdx} 4$ (a TF involved in embryonic hematopoiesis through 
activation of posterior Hox genes) alongside HoxB4 improved hematopoietic mesoderm specification as well as hematopoietic progenitor formation. The obtained HSPCs from this differentiation strategy can engraft more efficiently than strategies using only HoxB4 [56]. This finding demonstrates that the Cdx4-HoxB4 pathway is highly implicated in hematopoietic specification and formation, and its manipulation can potentially permit HSPC generation.

HOXB4 overexpression also leads to the generation of hematopoietic cells from human ESCs. Stable overexpression of HOXB4 permitted maintenance of cells in an undifferentiated state, and differentiation of these cells without cytokines demonstrated improved hematopoietic development. The addition of cytokines [stem cell factor (SCF), Fms-related tyrosine kinase 3 ligand (Flt3L), interleukin-3 (IL-3), IL-6, and granulocyte colony-stimulating factor (G-CSF)] that were previously established as important for hematopoietic expansion [57], however, further improved the yield of myeloid and monocytic colonies [58]. Despite this increased yield, another study found HOXB4 to be dispensable for hematopoietic development in human cells [59]. HOXB4 expression produces different effects in various cell types, and these effects depend on the timing of expression, amount of overexpression, and cellular environment. The apparent variability in HSPC function via HOXB4 reprogramming complicates our understanding of the role of HOXB4 in human hematopoiesis, and how to generate HSPCs capable of multilineage engraftment with this TF.

As a result of the difficulties with HoxB4, laboratories focused on other TFs as well as different hematopoietic cells derived from iPSCs, including lineage-restricted stem cells. Analysis of gene expression profiles revealed several underexpressed HSC-specific TFs in $\mathrm{CD} 34^{+} \mathrm{CD} 45^{+}$myeloid precursors as compared with $\mathrm{CD} 34^{+} \mathrm{CD} 38^{-} \mathrm{UCB}$ HSCs. Screening of these factors identified HOXA9, ERG, and RORA as genes capable of bestowing $\mathrm{CD} 34^{+} \mathrm{CD} 45^{+}$myeloid precursors with self-renewal and differentiation potential, but no engraftment potential. Screening of the few cells that did engraft revealed SOX4 and MYB as necessary factors to confer engraftment capability (Figure 2). These cells were used for short-term engraftment of myeloid and erythroid lineages [60]. The identification of these factors shows a possible regulatory gene network important for hematopoietic programming and engraftment. The erythroid cells generated from this strategy could be expanded exponentially and produced adult hemoglobin. This approach, however, suffers from the inability to develop cells with long-term engraftment potential and to generate lymphoid cells.

To more closely follow developmental hematopoiesis, human iPSCs were differentiated into HE with modified mRNA overexpression for select combinations of TFs that confer different lineage potentials. With ETV2 and GATA2, HE develops with subsequent generation of myeloid-biased hematopoietic cells. Transduction using GATA2 and TAL1 yields HE that generates hematopoietic cells with erythro-megakaryocytic lineage potential [61], demonstrating the importance of $\mathrm{HE}$ for the generation of hematopoietic cells. The inability to use one TF cocktail to induce cells with multilineage myeloid and lymphoid potential remains and the cells do not engraft immunodeficient mice long term. Although 
these cells closely follow developmental hematopoiesis by going through an HE stage, the inability to produce fully functional mature HSCs remains.

Further focusing on developmental hematopoiesis brings other TFs into play. Several recent studies have revealed that Sox 17 is crucial for HE development and definitive hematopoiesis by positively regulating NOTCH1 for both acquiring arterial fate as well as specifying HE that can undergo EHT via the Notch pathway [62-64]. Human PSC-derived HE with SOX17 overexpression forms semiadherent cell aggregates but gives rise to few hematopoietic progenitors, despite activation of several regulatory genes important in hematopoiesis. Upon release from SOX17 overexpression, however, these cells retain their hemogenic potential and give rise to many hematopoietic progenitors [65]. It remains unclear if these cells are capable of multilineage long-term engraftment. Other known TFs were shown to promote hematopoietic specification in PSCs. Overexpression of RUNX1a in EBs promoted hematopoietic commitment and definitive hematopoiesis, permitting hematopoietic progenitor cell generation. Upon RUNX1a overexpression, multiple mesoderm and hematopoietic genes are upregulated such as Brachyury and GATA2 [66]. SCL, a TF crucial for blood and endothelium specification from mesoderm during embryonic hematopoiesis, has an expression pattern that parallels hematopoietic specification in ESCs cultured with hematopoietic cytokines [57], and its overexpression increases hematopoietic specification even further. By contrast, SCL silencing substantially decreased the yield of hematopoietic progenitors [67].

All these TF studies in PSCs, although unable to generate HSPCs fully capable of multilineage engraftment, demonstrate the importance of several genes in hematopoiesis, and how their effects in overexpression studies are crucial for establishment of a hematopoietic program. Each study generates hematopoietic cells capable of various levels of function, demonstrating that a combination of several approaches and TFs could improve the function of the produced cells. Figure 2 presents a cartoon of the key studies with both mouse and human PSCs and Table 1 presents a synopsis of the various starting populations, factors, culture conditions, outcomes, and caveats to the technology.

\section{Reprogramming Somatic Cells to HSPCs}

Recent efforts have attempted to convert somatic cell fates without going through pluripotency [14], including studies to convert a somatic cell into the highly multipotent HSC. This section presents a synopsis of studies that have used pluripotency factors with hematopoietic TFs or miRNAs (Table 1, Figure 2).

\section{Inducing a State of Plasticity}

There is debate in the field as to whether introduction of pluripotency factors allows the induction of a plastic state such that, when alone or combined with other hematopoietic TFs and factors/culture conditions, HSPCs can be generated. It has been recently found, however, that when pluripotency factors are included in a reprogramming cocktail, a transient pluripotent intermediate is produced $[68,69]$. Whether or not a strategy attempts to avoid this stage, different studies have used pluripotency factors to make cell fate conversions more feasible with some success. 
Ectopic OCT4 overexpression and cytokine treatment generated $\mathrm{CD} 45^{+}$cells from human dermal fibroblasts (HDFs). These cells gave rise to various hematopoietic colonies (granulocytic, erythrocytic, monocytic, and megakaryotic) [70]. This method, however, generates cells with limited self-renewal potential that cannot produce lymphoid cells. Furthermore, engraftment was limited to the site of injection of the reprogrammed cells, making full hematopoietic reconstitution unlikely. Short-term exposure of OCT4 overexpressing adult HDFs to reprogramming media (RM) induced a state of plasticity, revealing the importance of the relationship between factor overexpression and extracellular environment, which affects the fibroblast transcriptome [71]. There are caveats to this methodology, as the introduction of OCT4 could generate plastic intermediates that may contaminate HSPC populations with partially pluripotent cells that are potentially tumorigenic.

In addition to the extracellular environment, overexpression of particular miRNAs can promote reprogramming to HSPCs. Indeed, overexpression of SOX2 in human fibroblasts helped rapidly form $\mathrm{CD} 34^{+}$cells, while expression of miR-125b assisted with engraftment potential. This strategy uses in situ development of their partially reprogrammed cells, relying on the hematopoietic niche of the mouse to complete reprogramming [16]. The use of the pluripotency factor SOX2, however, has the same potential issues as OCT4 [70,71]. Both genes could cause the production of partially pluripotent intermediates during this indirect conversion process. Furthermore, the cells generated via SOX2 and miR-125b overexpression in the presence of the mouse hematopoietic niche led only to the engraftment of cells that give rise to macrophage and monocyte-like progenitors.

\section{Inducing a Developmental Program In Vitro}

Using mouse embryonic fibroblasts (MEFs) from double transgenic mice that label CD34 ${ }^{+}$ cells with H2BGFP [72] to screen for appropriate TFs revealed that Gata2, Gfi1b, cFos, and Etv6 overexpression can induce a hemogenic program, closely recapitulating hematopoietic development during embryogenesis. Close inspection of the reprogramming process identified a putative precursor to $\mathrm{HE}$ that exhibited a Prominin $1{ }^{+} \mathrm{Sca} 1^{+} \mathrm{CD} 34^{+} \mathrm{CD} 45^{-}$cell surface phenotype and a global endothelial transcription program. Further culture led to the emergence of hematopoietic-like cells - possibly by budding from developing HE cells. These hematopoietic-like cells possessed an HSC transcriptional program and cell surface profile. After placental reaggregation culture, they adopted colony-forming potential in vitro [30]. This work establishes a platform to study developmental hematopoiesis in vitro. The discovery of an intermediate cell with a specific cell surface phenotype prompts the search to see if they exist in embryonic sites of emerging definitive hematopoiesis and if, when isolated, they can be matured into repopulating HSCs [73]. Work continues towards generating cells that have multilineage engraftment potential in mice and has been extended to human cells. It will be interesting to determine if the same TF cocktail induces a hemogenic program in human fibroblasts and/or if additional signals or factors are needed to develop HSCs with multilineage long-term engraftment potential.

Further demonstrating the establishment of an HE intermediate during reprogramming, Erg, Gata2, Lmo2, Runx1c, and Scl overexpression led to rapid derivation of hematopoietic 
progenitor cells within 8 days from embryonic and adult mouse fibroblasts. The derived cells travel through an HE intermediate, possessing both an endothelial and hematopoietic gene expression profile. These hematopoietic progenitors have multilineage potential (erythroid, megakaryocytic, and myeloid), and co-culture on OP9 and OP9-DL1 stroma allowed for the generation of lymphoid B and T cells, respectively, leading to short-term multilineage engraftment. Reprogramming $\mathrm{p}^{-1-}$ fibroblasts increased the efficiency and rapidity (5 days compared with 8 days) of derivation [74]. This approach mirrors the strategy using Gata2 and fibroblasts to reprogram to HSPCs through a hemogenic intermediate [30]. Although this report demonstrates rapid derivation of both precursors to hematopoietic cells and progenitors, loss of p53 may lead to untoward effects. Moreover, even with loss of p53, these cells only have short-term engraftment potential.

The TF cocktail Gata2, Lmo2, Mycn, Pitx2, Sox17, and Tal1 locks mouse cells (ESCs, fetal liver cells, and fibroblasts) in a proliferative self-renewing HB state called expandable HBs (eHBs). Continued expression of the TFs keeps the cells in a proliferative state but once the ectopic factors are silenced these eHBs give rise to functional smooth muscle, endothelial, and multi-lineage hematopoietic cells. The presence of fibroblast growth factor (FGF) in culture promotes expansion of these cells, and also supports the capability of eHBs to generate endothelial cells and leukocytes, but not erythrocytes [75]. Although it is encouraging that an expandable cell, in this case eHBs, can be generated, their multilineage engraftment potential was not assessed. Additionally, this process yielded poor results from fibroblasts, making it difficult to work in a patient-specific context.

\section{Direct Reprogramming together with Signals from the Microenvironment}

A very promising strategy has emerged where direct TF reprogramming together with inductive signals from the in vivo niche leads to the generation of bona fide mouse HSCs. Committed hematopoietic progenitor/effector cells with ectopic expression of Hlf, Runx1t1, Pbx1, Lmo2, Zfp37, and Prdm5 were immediately transplanted into mice with continued transgene expression for 2 weeks, allowing the in vivo niche to perform the reprogramming. Two additional factors, Meis1 and Mycn, along with polycistronic viruses, increased reprogramming efficiency [76]. Progenitor cells and differentiated cells from transplanted mice could be reprogrammed in vivo by turning on TFs after a subsequent transplant. While promising, this method is not without its caveats. The use of the in vivo niche precludes the ability to study the specific signals that are required to support HSC reprogramming. Additionally, several TFs in the reprogramming cocktail are proto-oncogenes, increasing the risk of oncogenesis. Furthermore, blood cells from patients suffering from hematopoietic disorders caused by either acquired or congenital mutations in the HSPC pool continue to bear these mutations when reprogrammed, making transplantation of these cell types in patients unfeasible. Nevertheless, it will be interesting to see if this strategy will work in human cells.

Another study found that reprogramming human umbilical vein and adult dermal microvascular endothelial cells into multipotent progenitors (MPPs) with the TFs FOSB, GFI1, RUNX1, and SPI1 (PU.1) required the support of an in vitro vascular niche. The reprogrammed MPPs engrafted primary and secondary immunodeficient mice, generating all 
cells of the hematopoietic lineage except for T cells [77]. The vascular niche layer of E4ECs (endothelial cells transduced with E4ORF1) was previously shown to be capable of expanding repopulating HSCs [78]. This cell line is thought to promote survival pathways without altering proliferation or transformation pathways, making the absence of serum possible. Other endothelial niche cells that overexpress the NOTCH ligands JAG1 and DLL4 induce PSC-derived MPPs to generate substantially more hematopoietic progenitors than does culturing these cells in the absence of this niche layer. These NOTCH ligands induce the expression of NOTCH targets RUNX1 and GATA2, both of which are crucial for definitive hematopoiesis [79]. This finding demonstrates that the activation of the NOTCH pathway during differentiation of PSCs is necessary for HSC emergence, and, together with the niche, plays a large role in developmental hematopoiesis. The long-term engraftment capability of reprogrammed endothelial cells into immunodeficient mice constitutes a step in the right direction for the field but the inability of these cells to generate T cells in vivo demonstrates that a complete multilineage hematopoietic program has not been established, necessitating future study to determine what inductive signals along with the E4EC layer may endow this capability. Furthermore, the use of endothelial cells can complicate the clinical application of this study resulting from the difficulty of obtaining sufficient numbers of patient endothelial cells for reprogramming.

\section{Concluding Remarks}

The ultimate goal of these studies is to generate expandable HSCs or progenitor populations that can be expanded in vitro for multiple uses. A few of these are as follows: (i) study hematopoiesis more fully in vitro; (ii) have appropriate disease modeling and drug testing systems for existing hematopoietic disorders; (iii) genetically correct and expand patientspecific HSCs with the intent of stem cell transplantation; and (iv) generate patient-specific blood products [80,81]. Although progress has been made, years of efforts have attempted in vitro/ex vivo expansion or the de novo generation of HSCs from PSCs, leaving much more work to be undertaken (see Outstanding Questions). Cell fate conversion of somatic cells to HSCs, although in its infancy, is an attractive alternative. It appears that somatic cell-derived HSPCs generated with the appropriate microenvironmental conditions functionally engraft more successfully than PSC-derived cells. Overall, we still need to determine ways to expand induced HSCs or precursor cells. The problems may lie in our lack of knowledge as to what inductive signals and microenvironmental cues are necessary to promote HSC expansion and maintain function.

\section{Outstanding Questions}

Which TF cocktail and culture condition (niche co-culture, cytokine supplementation, etc.) will allow for derivation of bona fide HSCs as defined as cells with long-term multilineage engraftment and self-renewal potential?

Will inclusion of miRNAs and epigenetic modifiers be required to augment TF reprogramming?

Will the use of TFs allow the development of PSC-derived HSCs with long-term multilineage engraftment and self-renewal potential? 
How do the molecular mechanisms of reprogramming vary across the different TF cocktails and starting cell populations?

How is chromatin and DNA modified during the reprogramming process?

Once reprogrammed HSPCs are generated will we be able to keep and expand them in culture?

Will it be possible to generate and expand intermediate cells that can subsequently turn into transplantable HSPCs?

Once bona fide definitive HSCs can faithfully be derived in vitro, what will be the most appropriate and applicable uses for this technology?

A variety of different TF combinations have been used in these studies, all with varying levels of success. The primary difference among the groups is the starting cell population (Table 1, Figure 2). Disparity between different TF cocktails could be attributable to a balance between instigating a hematopoietic program and repressing the cell identity of the starting population. Consistency among certain TFs such as GATA2 may identify the crucial need of this factor to induce a developmental program (either HE or HB) for various cell types such as fibroblasts or PSCs $[30,61,74,75]$. It is likely that key hematopoietic TFs such as RUNX1 will function in cells poised to generate HSPCs (such as HE cells) but will need to be turned on in other cell types as has been shown in fibroblasts going through the reprogramming process [30].

The methods by which TFs are introduced also add concern. Constitutive or conditional lentiviral expression remains the most common tool. Introduction of foreign DNA by this tool results in random genomic integration and may cause insertional mutagenesis and oncogenesis [82]. This concern is somewhat tempered by several successful gene therapy trials ongoing that use this technology [83]. This issue may be avoided by the use of modified mRNA or self-replicating mRNA technologies that have been previously shown to efficiently reprogram fibroblasts to iPSCs [84-86].

To date, the two most successful studies use starting cells (blood progenitors and endothelial cells) that are more epigenetically related to HSPCs than any other strategy [76,77], suggesting that the role of epigenetic closeness of the starting cell population or epigenetic memory of the reprogrammed cells must be considered. Low passage iPSCs retain DNA methylation signatures of their precursor cell type that can restrict differentiation, but can be reset upon further passaging $[87,88]$. This cell memory may thus inhibit function of the induced HSCs. Direct manipulation while reprogramming cells will be required to alter cell identity and overcome epigenetic memory [89]. These include the inductive signals of the microenvironment, as well as introduction of various agents such as small molecules to assist epigenetic reprogramming. Given what has been learned in recent years about the inductive signals of the niche as well as the impact of small molecules on HSPC expansion, the protocol that follows developmental hematopoiesis in vitro and incorporates these approaches will likely find the most success. 


\section{Acknowledgments}

Funding support provided by 1R01HL119404 for I.R.L. and K.A.M.

\section{Glossary}

\section{Allogeneic HSC transplant}

transplantation of bone marrow or isolated cells from peripheral blood from a donor that is then given to a host. There are major concerns of GvHD with this form of transplant

\section{Autologous HSC transplant}

transplantation of isolated cells (typically from UCB) belonging to the same person that requires the transplant. This type of transplant bypasses concerns related to HLA matching and GvHD. The abundance of required cells for transplant is the primary concern. Children require fewer HSCs than adults with regard to HSC transplant, thus UCB is useful primarily for children in need of transplant. Of course this is dependent upon whether the child's UCB has been banked

\section{Hemangioblast}

unlike the HE, the hemangioblast is defined as the theorized precursor cell to endothelium, blood cells, and smooth muscle cells

\section{Hematopoiesis}

the process by which an HSC gives rise in what is thought to be a hierarchical manner to every cell in the hematopoietic system. HSCs sit at the top of this hierarchy and give rise to progenitors that then divide into more lineage-restricted cells until they generate terminally differentiated cells such as leukocytes, macrophages, etc

\section{Hematopoietic stem cells (HSCs)}

can self-renew and differentiate down a hierarchy to form every terminally differentiated cell in the blood. A current major deficit in HSC investigative biology is our inability to culture HSCs long term, which currently hinders what we can study with them

\section{Hemogenic endothelium (HE)}

specialized endothelium theorized to give rise to HSCs via a process of cell budding. They are thought to be found in locations of embryonic definitive hematopoiesis such as the aortagonad-mesonephros region

\section{Multilineage long-term reconstitution/engraftment}

the ability of a cell with self-renewing HSC potential to repopulate the hematopoietic system of an irradiated or immunocompromised host for long periods of time and give rise to all the cells in the hematopoietic system. Self-renewal can be assessed in experimental conditions by transplanting marrow from the primary host into a secondary host

\section{Reprogramming}

the process where we introduce a stimulus (typically transduction with TFs, miRNAs, etc., as well as culture conditions) that will induce a fate change in the cell. Examples of 
reprogramming include the generation of iPSCs from somatic cells and the generation of other somatic cell types from a different somatic cell by a process of cell conversion

\section{Transcription factor}

protein that binds to specific regions of the DNA that can regulate transcription of DNA to mRNA

\section{References}

1. Takizawa H, et al. Ex vivo expansion of hematopoietic stem cells: mission accomplished? Swiss Med Wkly. 2011; 141:w13316. [PubMed: 22252776]

2. Siena S, et al. Therapeutic relevance of CD34 cell dose in blood cell transplantation for cancer therapy. J Clin Oncol. 2000; 18:1360-1377. [PubMed: 10715309]

3. Petersdorf EW. The major histocompatibility complex: a model for understanding graft-versus-host disease. Blood. 2013; 122:1863-1872. [PubMed: 23878143]

4. Boitano AE, et al. Aryl hydrocarbon receptor antagonists promote the expansion of human hematopoietic stem cells. Science. 2010; 329:1345-1348. [PubMed: 20688981]

5. Fares I, et al. Cord blood expansion. Pyrimidoindole derivatives are agonists of human hematopoietic stem cell self-renewal. Science. 2014; 345:1509-1512. [PubMed: 25237102]

6. Chaurasia P, et al. Epigenetic reprogramming induces the expansion of cord blood stem cells. J Clin Invest. 2014; 124:2378-2395. [PubMed: 24762436]

7. Liu ML, et al. Small molecules enable neurogenin 2 to efficiently convert human fibroblasts into cholinergic neurons. Nat Commun. 2013; 4:2183. [PubMed: 23873306]

8. Federation AJ, et al. The use of small molecules in somatic-cell reprogramming. Trends Cell Biol. 2014; 24:179-187. [PubMed: 24183602]

9. Zhang Y, et al. Small molecules, big roles - the chemical manipulation of stem cell fate and somatic cell reprogramming. J Cell Sci. 2012; 125:5609-5620. [PubMed: 23420199]

10. $\mathrm{Xu} \mathrm{Y,} \mathrm{et} \mathrm{al.} \mathrm{A} \mathrm{chemical} \mathrm{approach} \mathrm{to} \mathrm{stem-cell} \mathrm{biology} \mathrm{and} \mathrm{regenerative} \mathrm{medicine.} \mathrm{Nature.} \mathrm{2008;}$ 453:338-344. [PubMed: 18480815]

11. Takahashi K, Yamanaka S. Induction of pluripotent stem cells from mouse embryonic and adult fibroblast cultures by defined factors. Cell. 2006; 126:663-676. [PubMed: 16904174]

12. Takahashi $\mathrm{K}$, et al. Induction of pluripotent stem cells from adult human fibroblasts by defined factors. Cell. 2007; 131:861-872. [PubMed: 18035408]

13. Lee TI, Young RA. Transcriptional regulation and its misregulation in disease. Cell. 2013; 152:1237-1251. [PubMed: 23498934]

14. Pereira CF, et al. Reprogramming cell fates: insights from combinatorial approaches. Ann NY Acad Sci. 2012; 1266:7-17. [PubMed: 22901251]

15. Orkin SH. Transcription factors and hematopoietic development. J Biol Chem. 1995; 270:49554958. [PubMed: 7890597]

16. Pulecio J, et al. Conversion of human fibroblasts into monocyte-like progenitor cells. Stem Cells. 2014; 32:2923-2938. [PubMed: 25175072]

17. Kurian L, et al. Conversion of human fibroblasts to angioblast-like progenitor cells. Nat Methods. 2013; 10:77-83. [PubMed: 23202434]

18. Vierbuchen T, et al. Direct conversion of fibroblasts to functional neurons by defined factors. Nature. 2010; 463:1035-1041. [PubMed: 20107439]

19. Sekiya S, Suzuki A. Direct conversion of mouse fibroblasts to hepatocyte-like cells by defined factors. Nature. 2011; 475:390-393. [PubMed: 21716291]

20. Qian L, et al. In vivo reprogramming of murine cardiac fibroblasts into induced cardiomyocytes. Nature. 2012; 485:593-598. [PubMed: 22522929]

21. Choi J, et al. MyoD converts primary dermal fibroblasts, chondroblasts, smooth muscle, and retinal pigmented epithelial cells into striated mononucleated myoblasts and multinucleated myotubes. Proc Natl Acad Sci USA. 1990; 87:7988-7992. [PubMed: 2172969] 
22. Davis RL, et al. Expression of a single transfected cDNA converts fibroblasts to myoblasts. Cell. 1987; 51:987-1000. [PubMed: 3690668]

23. Son EY, et al. Conversion of mouse and human fibroblasts into functional spinal motor neurons. Cell Stem Cell. 2011; 9:205-218. [PubMed: 21852222]

24. Ieda M, et al. Direct reprogramming of fibroblasts into functional cardiomyocytes by defined factors. Cell. 2010; 142:375-386. [PubMed: 20691899]

25. Buganim Y, et al. Direct reprogramming of fibroblasts into embryonic Sertoli-like cells by defined factors. Cell Stem Cell. 2012; 11:373-386. [PubMed: 22958931]

26. Feng R, et al. PU. 1 and $\mathrm{C} / \mathrm{EBP} \propto / \beta$ convert fibroblasts into macrophage-like cells. Proc Natl Acad Sci USA. 2008; 105:6057-6062. [PubMed: 18424555]

27. Han DW, et al. Direct reprogramming of fibroblasts into neural stem cells by defined factors. Cell Stem Cell. 2012; 10:465-472. [PubMed: 22445517]

28. Huang $\mathrm{P}$, et al. Induction of functional hepatocyte-like cells from mouse fibroblasts by defined factors. Nature. 2011; 475:386-389. [PubMed: 21562492]

29. Najm FJ, et al. Transcription factor-mediated reprogramming of fibroblasts to expandable, myelinogenic oligodendrocyte progenitor cells. Nat Biotechnol. 2013; 31:426-433. [PubMed: 23584611]

30. Pereira CF, et al. Induction of a hemogenic program in mouse fibroblasts. Cell Stem Cell. 2013; 13:205-218. [PubMed: 23770078]

31. Zhou Q, Melton DA. Extreme makeover: converting one cell into another. Cell Stem Cell. 2008; 3:382-388. [PubMed: 18940730]

32. Robinton DA, Daley GQ. The promise of induced pluripotent stem cells in research and therapy. Nature. 2012; 481:295-305. [PubMed: 22258608]

33. Papapetrou EP, Sadelain M. Reconstructing blood from induced pluripotent stem cells. F1000 Med Rep. 2010; 2:44. [PubMed: 20948840]

34. Murry CE, Keller G. Differentiation of embryonic stem cells to clinically relevant populations: lessons from embryonic development. Cell. 2008; 132:661-680. [PubMed: 18295582]

35. Kim PG, Daley GQ. Application of induced pluripotent stem cells to hematologic disease. Cytotherapy. 2009; 11:980-989. [PubMed: 19929461]

36. Lim WF, et al. Hematopoietic cell differentiation from embryonic and induced pluripotent stem cells. Stem Cell Res Ther. 2013; 4:71. [PubMed: 23796405]

37. Keller G, et al. Hematopoietic commitment during embryonic stem cell differentiation in culture. Mol Cell Biol. 1993; 13:473-486. [PubMed: 8417345]

38. Hole N, et al. A limited temporal window for the derivation of multilineage repopulating hematopoietic progenitors during embryonal stem cell differentiation in vitro. Blood. 1996; 88:1266-1276. [PubMed: 8695844]

39. Choi KD, et al. Identification of the hemogenic endothelial progenitor and its direct precursor in human pluripotent stem cell differentiation cultures. Cell Rep. 2012; 2:553-567. [PubMed: 22981233]

40. Zovein AC, et al. Fate tracing reveals the endothelial origin of hematopoietic stem cells. Cell Stem Cell. 2008; 3:625-636. [PubMed: 19041779]

41. Bertrand JY, et al. Haematopoietic stem cells derive directly from aortic endothelium during development. Nature. 2010; 464:108-111. [PubMed: 20154733]

42. Gordon-Keylock S, Medvinsky A. Endothelio-hematopoietic relationship: getting closer to the beginnings. BMC Biol. 2011; 9:88. [PubMed: 22204560]

43. Chen MJ, et al. Erythroid/myeloid progenitors and hematopoietic stem cells originate from distinct populations of endothelial cells. Cell Stem Cell. 2011; 9:541-552. [PubMed: 22136929]

44. Antas VI, et al. Hemogenic endothelium: a vessel for blood production. Int J Biochem Cell Biol. 2013; 45:692-695. [PubMed: 23270729]

45. Chen MJ, et al. Runx1 is required for the endothelial to haematopoietic cell transition but not thereafter. Nature. 2009; 457:887-891. [PubMed: 19129762]

46. Sturgeon CM, et al. Defining the path to hematopoietic stem cells. Nat Biotechnol. 2013; 31:416418. [PubMed: 23657396]

Trends Cell Biol. Author manuscript; available in PMC 2017 March 01. 
47. Boiers $\mathrm{C}$, et al. Lymphomyeloid contribution of an immune-restricted progenitor emerging prior to definitive hematopoietic stem cells. Cell Stem Cell. 2013; 13:535-548. [PubMed: 24054998]

48. Lancrin $\mathrm{C}$, et al. The haemangioblast generates haematopoietic cells through a haemogenic endothelium stage. Nature. 2009; 457:892-895. [PubMed: 19182774]

49. Amabile $\mathrm{G}$, et al. In vivo generation of transplantable human hematopoietic cells from induced pluripotent stem cells. Blood. 2013; 121:1255-1264. [PubMed: 23212524]

50. Takaki S, et al. Enhanced hematopoiesis by hematopoietic progenitor cells lacking intracellular adaptor protein, Lnk. J Exp Med. 2002; 195:151-160. [PubMed: 11805142]

51. Suzuki N, et al. Generation of engraftable hematopoietic stem cells from induced pluripotent stem cells by way of teratoma formation. Mol Ther. 2013; 21:1424-1431. [PubMed: 23670574]

52. Forrester LM, Jackson M. Mechanism of action of HOXB4 on the hematopoietic differentiation of embryonic stem cells. Stem Cells. 2012; 30:379-385. [PubMed: 22267295]

53. McKinney-Freeman SL, et al. Isolation of hematopoietic stem cells from mouse embryonic stem cells. Curr Protoc Stem Cell Biol. 2008; (Suppl 4):1F.3.1-1F.3.10.

54. Kyba M, et al. HoxB4 confers definitive lymphoid-myeloid engraftment potential on embryonic stem cell and yolk sac hematopoietic progenitors. Cell. 2002; 109:29-37. [PubMed: 11955444]

55. Tashiro K, et al. Promotion of hematopoietic differentiation from mouse induced pluripotent stem cells by transient HoxB4 transduction. Stem Cell Res. 2012; 8:300-311. [PubMed: 22000550]

56. Wang Y, et al. Embryonic stem cell-derived hematopoietic stem cells. Proc Natl Acad Sci USA. 2005; 102:19081-19086. [PubMed: 16357205]

57. Chadwick K, et al. Cytokines and BMP-4 promote hematopoietic differentiation of human embryonic stem cells. Blood. 2003; 102:906-915. [PubMed: 12702499]

58. Bowles KM, et al. HOXB4 overexpression promotes hematopoietic development by human embryonic stem cells. Stem Cells. 2006; 24:1359-1369. [PubMed: 16410392]

59. Wang L, et al. Generation of hematopoietic repopulating cells from human embryonic stem cells independent of ectopic HOXB4 expression. J Exp Med. 2005; 201:1603-1614. [PubMed: 15883170]

60. Doulatov S, et al. Induction of multipotential hematopoietic progenitors from human pluripotent stem cells via respecification of lineage-restricted precursors. Cell Stem Cell. 2013; 13:459-470. [PubMed: 24094326]

61. Elcheva I, et al. Direct induction of haematoendothelial programs in human pluripotent stem cells by transcriptional regulators. Nat Commun. 2014; 5:4372. [PubMed: 25019369]

62. Clarke RL, et al. The expression of Sox 17 identifies and regulates haemogenic endothelium. Nat Cell Biol. 2013; 15:502-510. [PubMed: 23604320]

63. Ditadi A, et al. Human definitive haemogenic endothelium and arterial vascular endothelium represent distinct lineages. Nat Cell Biol. 2015; 17:580-591. [PubMed: 25915127]

64. Corada M, et al. Sox17 is indispensable for acquisition and maintenance of arterial identity. Nat Commun. 2013; 4:2609. [PubMed: 24153254]

65. Nakajima-Takagi Y, et al. Role of SOX17 in hematopoietic development from human embryonic stem cells. Blood. 2013; 121:447-458. [PubMed: 23169777]

66. Ran D, et al. RUNX1a enhances hematopoietic lineage commitment from human embryonic stem cells and inducible pluripotent stem cells. Blood. 2013; 121:2882-2890. [PubMed: 23372166]

67. Real PJ, et al. SCL/TAL1 regulates hematopoietic specification from human embryonic stem cells. Mol Ther. 2012; 20:1443-1453. [PubMed: 22491213]

68. Bar-Nur O, et al. Lineage conversion induced by pluripotency factors involves transient passage through an iPSC stage. Nat Biotechnol. 2015; 33:761-768. [PubMed: 26098450]

69. Maza I, et al. Transient acquisition of pluripotency during somatic cell transdifferentiation with iPSC reprogramming factors. Nat Biotechnol. 2015; 33:769-774. [PubMed: 26098448]

70. Szabo E, et al. Direct conversion of human fibroblasts to multilineage blood progenitors. Nature. 2010; 468:521-526. [PubMed: 21057492]

71. Mitchell R, et al. Molecular evidence for OCT4-induced plasticity in adult human fibroblasts required for direct cell fate conversion to lineage specific progenitors. Stem Cells. 2014; 32:2178 2187. [PubMed: 24740884] 
72. Qiu J, et al. Divisional history and hematopoietic stem cell function during homeostasis. Stem Cell Rep. 2014; 2:473-490.

73. Pereira CF, et al. There will be blood" from fibroblasts. Cell Cycle. 2014; 13:335-336. [PubMed: 24335410]

74. Batta K, et al. Direct reprogramming of murine fibroblasts to hematopoietic progenitor cells. Cell Rep. 2014; 9:1871-1884. [PubMed: 25466247]

75. Vereide DT, et al. An expandable, inducible hemangioblast state regulated by fibroblast growth factor. Stem Cell Rep. 2014; 3:1043-1057.

76. Riddell J, et al. Reprogramming committed murine blood cells to induced hematopoietic stem cells with defined factors. Cell. 2014; 157:549-564. [PubMed: 24766805]

77. Sandler VM, et al. Reprogramming human endothelial cells to haematopoietic cells requires vascular induction. Nature. 2014; 511:312-318. [PubMed: 25030167]

78. Butler JM, et al. Development of a vascular niche platform for expansion of repopulating human cord blood stem and progenitor cells. Blood. 2012; 120:1344-1347. [PubMed: 22709690]

79. Gori JL, et al. Vascular niche promotes hematopoietic multipotent progenitor formation from pluripotent stem cells. J Clin Invest. 2015; 125:1243-1254. [PubMed: 25664855]

80. Vierbuchen T, Wernig M. Direct lineage conversions: unnatural but useful? Nat Biotechnol. 2011; 29:892-907. [PubMed: 21997635]

81. Cherry AB, Daley GQ. Reprogrammed cells for disease modeling and regenerative medicine. Annu Rev Med. 2013; 64:277-290. [PubMed: 23327523]

82. Sadelain M. Insertional oncogenesis in gene therapy: how much of a risk? Gene Ther. 2004; 11:569-573. [PubMed: 15029226]

83. Persons DA. Lentiviral vector gene therapy: effective and safe? Mol Ther. 2010; 18:861-862. [PubMed: 20436489]

84. Mandal PK, Rossi DJ. Reprogramming human fibroblasts to pluripotency using modified mRNA. Nat Protoc. 2013; 8:568-582. [PubMed: 23429718]

85. Warren L, et al. Highly efficient reprogramming to pluripotency and directed differentiation of human cells with synthetic modified mRNA. Cell Stem Cell. 2010; 7:618-630. [PubMed: 20888316]

86. Yoshioka N, et al. Efficient generation of human iPSCs by a synthetic self-replicative RNA. Cell Stem Cell. 2013; 13:246-254. [PubMed: 23910086]

87. Vaskova EA, et al. Epigenetic memory" phenomenon in induced pluripotent stem cells. Acta Nat. 2013; 5:15-21.

88. Kim K, et al. Epigenetic memory in induced pluripotent stem cells. Nature. 2010; 467:285-290. [PubMed: 20644535]

89. Papp B, Plath K. Reprogramming to pluripotency: stepwise resetting of the epigenetic landscape. Cell Res. 2011; 21:486-501. [PubMed: 21321600] 


\section{Trends}

Many reprogramming strategies attempt to derive hematopoietic stem and progenitor cells (HSPCs) de novo from pluripotent stem cells (PSCs) or somatic cells. Each strategy yields hematopoietic cells of varying functionality.

The in vivo or in vitro niche, cytokine supplementation, and culture media greatly influence reprogramming efficiency. Incorporating these elements into a finalized reprogramming protocol is crucial to generate bona fide HSCs.

Some reprogramming strategies recapitulate developmental hematopoiesis 'in a dish'. This allows us to study blood development in vitro as well as the pathways involved in hematologic disease.

Once perfected, HSPC reprogramming protocols will be used for hematologic disease modeling and drug discovery. Also, patient-specific HSPC transplant will circumvent the risk of graft-versus-host disease and other immunological complications. 


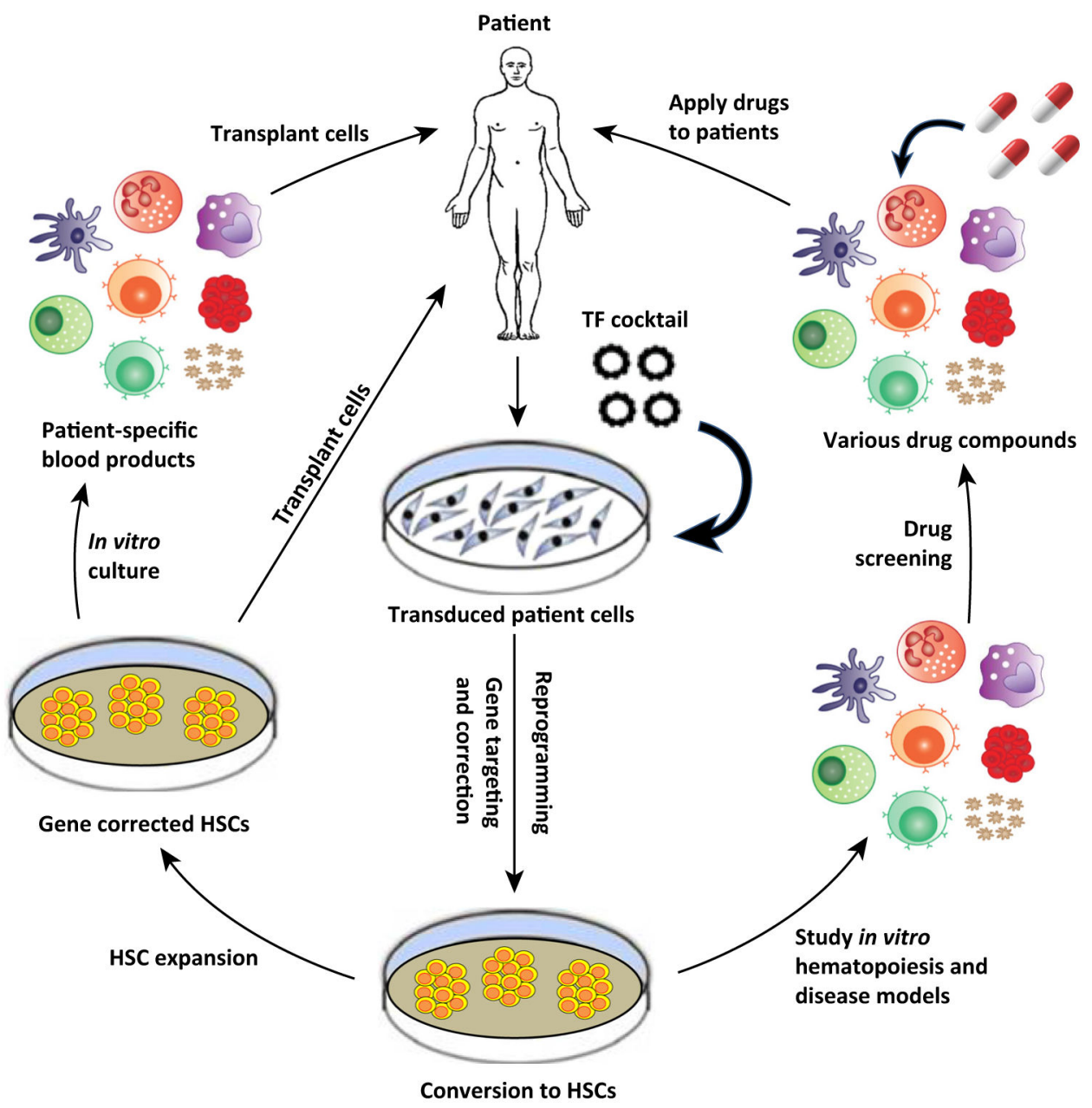

Figure 1.

Patient-Specific Hematopoietic Stem and Progenitor Cell (HSPC) Derivation and Future Studies. This diagram demonstrates the general strategy of most patient-specific cell reprogramming processes and future directions. The ideal strategy is to obtain patient/donor somatic cells and reprogram to the cell type of choice, in this case hematopoietic stem cells (HSCs). These HSCs could then be used in a variety of different studies. These include but are not limited to, gene correcting the derived HSCs (or correcting the genetic defect in the obtained patient cells before reprogramming), transplantation, drug screens to identify novel therapeutics for a variety of diseases, generating patient-specific blood products and studying hematopoiesis in vitro. 


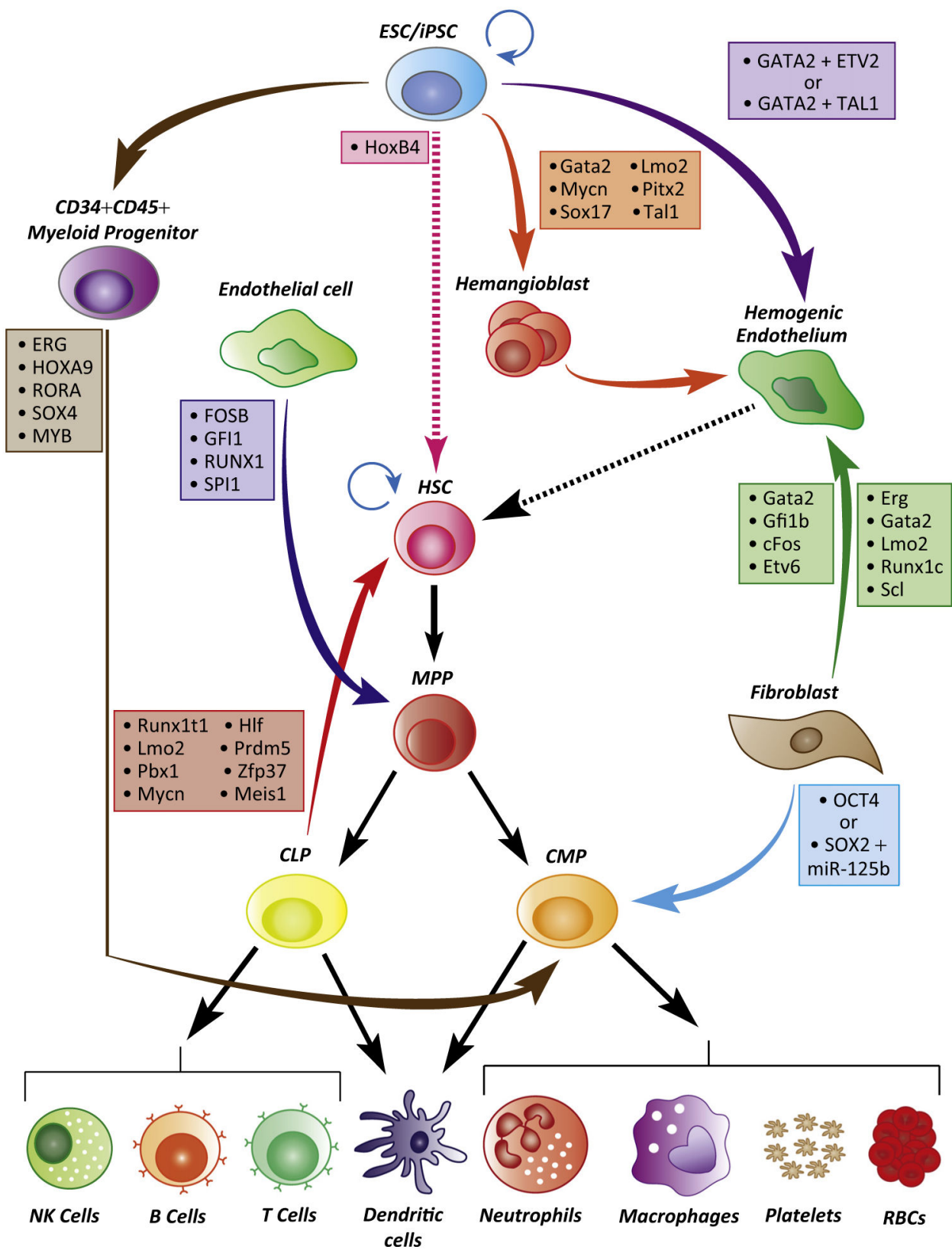

Figure 2.

Various Strategies to Generate Hematopoietic Stem Cells (HSCs). Several groups have attempted to derive HSCs in many different ways. The major differences among the strategies are the starting cells [embryonic stem cells (ESCs), induced pluripotent stem cells (iPSCs), fibroblasts, lineage committed blood progenitors, and endothelial cells], the media/ culture system the cells are reprogrammed on, and the transcription factor (TF) cocktail that the cells are subjected to. Although all efforts are aimed at getting bona fide HSCs, most of the attempts thus far fall short. The boxes list TFs and they are color coded to the arrow denoting the associated outcome. 


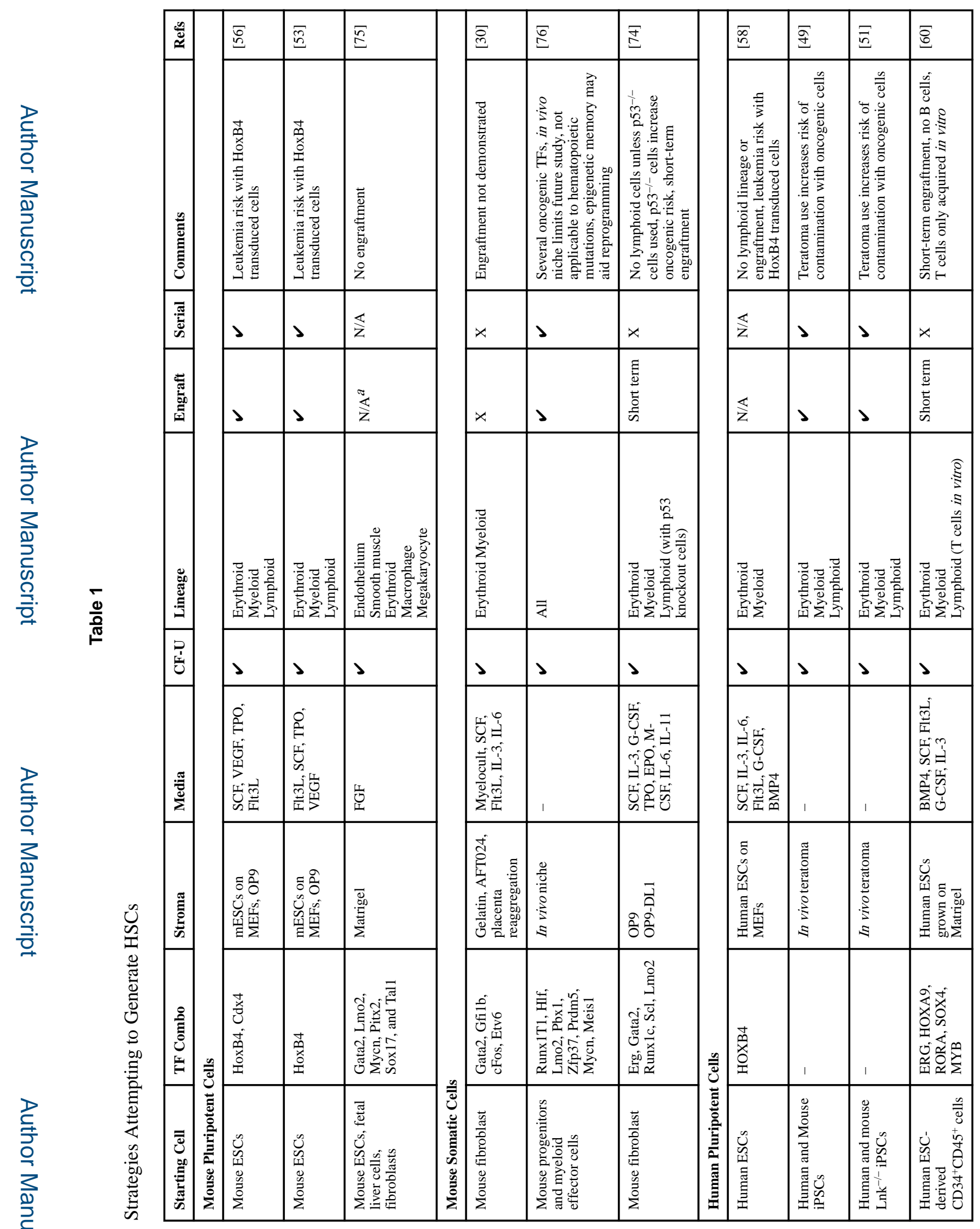

Trends Cell Biol. Author manuscript; available in PMC 2017 March 01. 


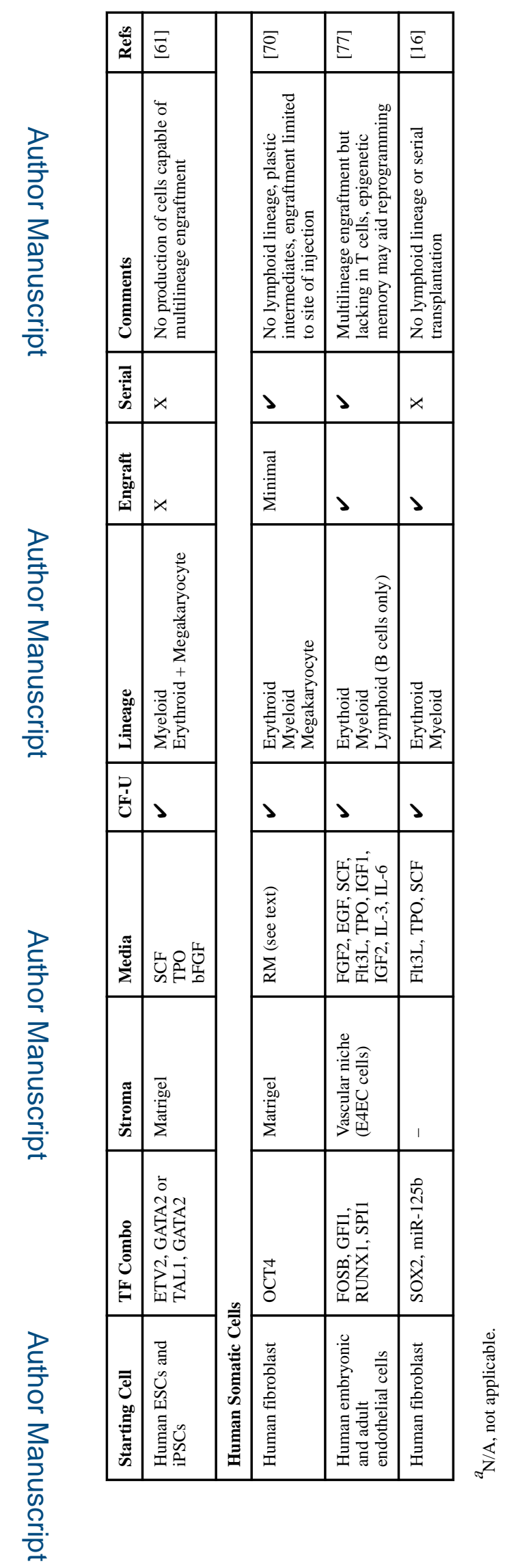

Trends Cell Biol. Author manuscript; available in PMC 2017 March 01. 